\title{
Inactivation of fatty acid transport protein 1 prevents fat-induced insulin resistance in skeletal muscle
}

\author{
Jason K. Kim, ${ }^{1}$ Ruth E. Gimeno, ${ }^{2}$ Takamasa Higashimori, ${ }^{1}$ Hyo-Jeong Kim, ${ }^{1}$ Hyejeong Choi, ${ }^{1}$ \\ Sandhya Punreddy, ${ }^{2}$ Robin L. Mozell, ${ }^{2}$ Guo Tan, ${ }^{2}$ Alain Stricker-Krongrad, ${ }^{2}$ David J. Hirsch, ${ }^{3,4}$ \\ Jonathan J. Fillmore, ${ }^{1}$ Zhen-Xiang Liu, ${ }^{1}$ Jianying Dong, ${ }^{1}$ Gary Cline, ${ }^{1}$ Andreas Stahl, 5 \\ Harvey F. Lodish, ${ }^{3,4}$ and Gerald I. Shulman ${ }^{1,6,7}$
}

\begin{abstract}
${ }^{1}$ Department of Internal Medicine, Yale University School of Medicine, New Haven, Connecticut, USA. 2Millennium Pharmaceuticals Inc., Cambridge, Massachusetts, USA. ${ }^{3}$ Whitehead Institute for Biomedical Research, Cambridge, Massachusetts, USA. ${ }^{4}$ Department of Biology, Massachusetts Institute of Technology, Cambridge, Massachusetts, USA. ${ }^{5}$ Research Institute, Palo Alto Medical Foundation, Palo Alto, California, USA. ${ }^{6}$ Department of Cellular and Molecular Physiology and 7The Howard Hughes Medical Institute, Yale University School of Medicine, New Haven, Connecticut, USA.
\end{abstract}

\begin{abstract}
Insulin resistance in skeletal muscle plays a major role in the development of type 2 diabetes and may be causally associated with increases in intramuscular fatty acid metabolites. Fatty acid transport protein 1 (FATP1) is an acylCoA synthetase highly expressed in skeletal muscle and modulates fatty acid uptake and metabolism by converting fatty acids into fatty acyl-CoA. To investigate the role of FATP1 in glucose homeostasis and in the pathogenesis of insulin resistance, we examined the effect of acute lipid infusion or chronic high-fat feeding on insulin action in FATP1 KO mice. Whole-body adiposity, adipose tissue expression of adiponectin, intramuscular fatty acid metabolites, and insulin sensitivity were not altered in FATP1 KO mice fed a regular chow diet. In contrast, FATP1 deletion protected the $\mathrm{KO}$ mice from fat-induced insulin resistance and intramuscular accumulation of fatty acylCoA without alteration in whole-body adiposity. These findings demonstrate an important role of intramuscular fatty acid metabolites in causing insulin resistance and suggest that FATP1 may be a novel therapeutic target for the treatment of insulin resistance and type 2 diabetes.
\end{abstract}

\section{Introduction}

Type 2 diabetes mellitus associated with obesity is reaching epidemic proportions in the world (1). Insulin resistance in skeletal muscle plays a primary role in its pathogenesis, yet the underlying factors causing insulin resistance remain unknown (2-4). Numerous factors have been implicated in this process, including circulating adipocyte-derived factors (i.e., resistin, adiponectin, leptin, TNF- $\alpha$ ) (5-9) and fatty acids (10-15). Previous studies have demonstrated increased expression of resistin and TNF- $\alpha$ or reduced expression of adiponectin and leptin to promote insulin resistance (5-9). Other studies have shown a strong inverse relationship between intramuscular fat content and insulin sensitivity in both animal models and humans (16-19). Increasing fatty acid delivery into skeletal muscle by overexpressing lipoprotein lipase caused insulin resistance (17), while decreasing fatty acid uptake into skeletal muscle with CD36 deletion improved insulin sensitivity (20). While the mechanism by which increased fatty acid levels cause insulin resistance remains inconclusive, our recent study suggested that intracellular accumulation of fatty acid-derived metabolites (i.e., fatty acyl-CoA, diacylglycerol) blunted insulin signaling activities and may be causally involved in the pathogenesis of fat-induced insulin resistance in skeletal muscle (21).

Nonstandard abbreviations used: $2-\left[{ }^{14} \mathrm{C}\right] \mathrm{DG}-6$-phosphate (2-DG-6-P); 2-deoxy-D-[1-14 C]glucose (2-[14C]DG); fatty acid-binding protein (FABP); fatty acid transport protein-1 (FATP1); hepatic glucose production (HGP); IкB kinase- $\beta$ (IKK- $\beta$ ); insulin receptor substrate-1 (IRS-1).

Conflict of interest: R.E. Gimeno, S. Punreddy, R.L. Mozell, G. Tan, and A. StrickerKrongrad were employees at Millennium at the time the work was done and had stock options in Millennium. None of these authors is currently employed by Millennium. Citation for this article: J. Clin. Invest. 113:756-763 (2004). doi:10.1172/JCI200418917.
Fatty acid transport protein 1 (FATP1) is a 646-amino acid integral plasma membrane protein that is expressed in cells and tissues with high levels of fatty acid uptake for metabolism or storage, such as skeletal muscle, adipose tissue, and the heart (22-24). Overexpression of mouse FATP1 in cultured fibroblasts resulted in a marked increase in long-chain fatty acid uptake (22), whereas disruption of the yeast Saccharomyces cerevisiae FATP1 homologue fat 1 resulted in impaired long-chain fatty acid uptake (25). Since purified FATP1 has acyl-CoA synthetase activity (26), increased fatty acid uptake mediated by FATP1 overexpression is likely driven by increased conversion of fatty acids to fatty acyl-CoA. Consistent with this notion, mutation of a key residue that is predicted to abolish catalytic activity results in a loss of FATP1-mediated fatty acid uptake (27). Fatty acids taken up through FATP1 are preferentially channeled into triglyceride synthesis, suggesting a functional link between FATP1-mediated fatty acid uptake and lipid storage (28).

In mammals, FATPs form a family of six related proteins. FATP4 is most closely related to FATP1 and is highly expressed in skeletal muscle, adipose tissue, and the heart (29). Deletion of FATP4 resulted in early embryonic (30) or neonatal lethality with a phenotype reminiscent of lethal restrictive dermopathy (31). Since both FATP1 and FATP4 are upregulated during adipocyte differentiation, and FATP1, but not FATP4, translocates to the plasma membrane in response to insulin, FATP1 may be involved in the hormonal regulation of fatty acid uptake, while FATP4 may mediate basal fatty acid uptake (32). FATP2, FATP3, FATP5, and FATP6 are more distally related to FATP1 and show little or no expression in adipose tissue and muscle $(29,33)$. FATP6 is specifically expressed in the heart and is the predominant FATP family member in this organ (33). While FATP3 is broadly expressed, FATP2 
and FATP5 show highly restricted tissue distribution in the liver (FATP2, FATP5) and kidney (FATP2) (29).

In addition to the FATPs, other proteins, such as long-chain acylCoA synthases (e.g., ACS1), the scavenger receptor family member fatty acid translocase/CD36, and members of the fatty acid-binding protein (FABP) family have also been implicated in playing a role in fatty acid uptake into cells $(22,23,34,35)$. Studies in yeast and mammalian cells suggest that FATPs and ACS1 can partly substitute for one another and form a multiprotein complex on the plasma membrane $(22,36)$. CD36 has been proposed to mediate fatty acid uptake by binding and thus increasing the local concentration of fatty acids at the plasma membrane (29). The importance of CD36 in fatty acid metabolism has been demonstrated in previous studies demonstrating that transgenic mice overexpressing CD36 have increased fatty acid uptake and oxidation $(20,37,38)$. Additionally, Hajri et al. have shown that CD36-null mice had reduced fatty acid uptake and enhanced insulin sensitivity, which implicates an important role of fatty acid transport proteins in whole-body glucose homeostasis (20).

In light of these findings, we have generated FATP1 KO mice to determine the role of FATP1 in fatty acid metabolism and glucose homeostasis in vivo. Furthermore, the effect of acute lipid infusion or chronic high-fat feeding on insulin action and signaling in the skeletal muscle of FATP1 KO mice was examined to determine the role of intramuscular accumulation of fatty acid-derived metabolites in the pathogenesis of fat-induced insulin resistance in skeletal muscle.

\section{Methods}

Generation of FATP1 KO mice. Genomic DNA containing the FATP1 locus was obtained by screening a 129/Sv genomic bacterial artificial chromosome library (Research Genetics, Huntsville, Alabama, USA) with a fragment containing the $5^{\prime}$ end of the FATP1 coding sequence. A targeting construct was generated by PCR amplifying $2.3 \mathrm{~kb}$ and 2 $\mathrm{kb}$ of genomic DNA immediately upstream and downstream of the first coding exon (see GenBank accession numbers AF023256-8 for the genomic structure of FATP1) and inserting these fragments into a targeting vector containing a neomycin resistance marker under control of the phosphoglycerate kinase-promoter (PGK-neo) and a lacz gene with a nuclear localization sequence ( $n l s-l a c z)$ located immediately downstream of the $5^{\prime}$ arm, thus placing lacz under the transcriptional control of the FATP1 promoter. To allow confirmation of recombination events, probes were generated by PCR from genomic DNA immediately adjacent to the $5^{\prime}$ and $3^{\prime}$ arms. FATP1 KO mice were generated by transfecting $129 \mathrm{SvEv}$ ES cells with the targeting construct, and ES cell clones that had undergone homologous recombination were injected into blastocysts that were then transferred to pseudopregnant female mice to generate chimeric offspring. Male chimeras were mated with $\mathrm{C} 57 \mathrm{Bl} / 6 \mathrm{~J}$ females to obtain germline transmission of the disrupted FATP1 gene. The resulting heterozygotes were interbred to generate mice homozygous for the FATP1 mutation, along with wild-type littermates. Genotyping of offspring was performed using a PCR assay with primers designed against the $5^{\prime}$ untranslated region of FATP1 (5'-GGCGGGCAATGTTAAGTAAACTGG-3'), sequences in the first coding exon ( $5^{\prime}$-CTCACACCACCCTGCAAGACTCT- $\left.3^{\prime}\right)$ to detect the WT allele, and sequences in the inserted cassette (5'-GTAATGGGATAGGTCACGTTGGTG$\left.3^{\prime}\right)$ to detect the $\mathrm{KO}$ allele.

Examination of gene expression. The mRNA expression of FATP family members, adiponectin and resistin, was performed using real-time quantitative PCR with an ABI Prism 7700 sequence detector (PerkinElmer Applied Biosystems Inc., Foster City, California, USA) accord- ing to the manufacturer's recommendations. RNA was prepared from homogenized tissues using TRIzol, treated with DNase A, and then converted to cDNA using reverse transcriptase with random hexamer and oligo-dT primers. To normalize the expression level of target genes 18S was used as an endogenous control. Primers and probes were designed using Primer Express software (Perkin-Elmer Applied Biosystems Inc.). Values are expressed as relative levels. To determine gene expression changes at the genome level, Affymetrix GeneChip technology was used (Affymetrix Inc., Santa Clara, California, USA). Samples were labeled and hybridized to the GeneChip Mouse Expression Set 430 following the manufacturer's protocols. The arrays were scanned on an Affymetrix GeneArray scanner. Data analysis was performed using MAS 5.0 software.

Metabolic phenotyping of FATP1 KO mice. Fourteen-week-old age- and sex-matched FATP1 KO and WT littermates were placed on a standard lab chow or a high-fat diet (58\% fat by calories, diet D12330; Research Diets Inc., New Brunswick, New Jersey, USA). Body weight was assessed weekly. Fat and lean masses were determined after 11 weeks on the diet by dual-energy $\mathrm{x}$-ray absorptiometry using a PIXImus mouse densitometer (Lunar Corp., Madison, Wisconsin, USA). Fourteen weeks after initiation of the diet, animals were fasted overnight, glucose was measured using a Glucometer (Bayer Corp., Elkhard, Indiana, USA), and plasma and tissues were collected. Commercially available kits were used to determine plasma insulin, (Crystal Chem Inc., Downers Grove, Illinois, USA), free fatty acids, and triglycerides (both from SigmaAldrich, St. Louis, Missouri, USA). All procedures were approved by the Millennium Institutional Animal Care and Use Committee.

Hyperinsulinemic-englycemic clamps to assess insulin action and signaling in vivo. Male FATP1 KO and WT littermates weighing approximately $25 \mathrm{~g}$ (approximately 16 weeks of age) were studied to examine the effect of acute lipid infusion or chronic high-fat feeding on insulin action and signaling. For the chronic high-fat feeding study, WT $(n$ $=6)$ and FATP1 KO $(n=5)$ mice were fed ad libitum with a high-fat $\operatorname{diet}$ (55\% fat, no. 93075; Harlan Teklad, Madison, Wisconsin, USA) for 3 weeks prior to the in vivo experiments. At least 4 days before hyperinsulinemic-euglycemic clamp experiments, an indwelling catheter was inserted in the right internal jugular vein of mice as previously described (17). All procedures were approved by the Yale University Animal Care and Use Committee.

After an overnight fast, HPLC-purified $\left[3-{ }^{3} \mathrm{H}\right]$ glucose $(0.05$ $\mu \mathrm{Ci} / \mathrm{min}$; Amersham Biosciences, Piscataway, New Jersey, USA) was infused for 2 hours (basal period) to estimate the rate of basal glucose turnover. Following the basal period, a 2-hour hyperinsulinemic-euglycemic clamp experiment was conducted with a primed, continuous infusion of human insulin (15 pmol/kg/min) (Humulin; Eli Lilly and Co., Indianapolis, Indiana, USA) to raise plasma insulin levels, while plasma glucose was maintained at basal concentrations with variable rates of $20 \%$ glucose infusion. Insulin-stimulated whole-body glucose metabolism rates were estimated with a continuous infusion of $\left[3-{ }^{3} \mathrm{H}\right]$ glucose $(0.1 \mu \mathrm{Ci} / \mathrm{min})$ during the clamp procedure, and 2-deoxy-D-[1-14C]glucose $\left(2-\left[{ }^{14} \mathrm{C}\right] \mathrm{DG}\right.$; Amersham Biosciences) was bolus injected at the 75 -minute time point of the clamp procedure to estimate the rates of insulin-stimulated tissue-specific glucose uptake as previously described (17). At the end of the clamp procedure, tissues were taken for biochemical measurements. For the acute lipid infusion experiments, lipid $(5 \mathrm{ml} / \mathrm{kg} / \mathrm{h}$, Liposyn II; the triglyceride emulsion was $20 \% \mathrm{wt} / \mathrm{vol}$ ) (Abbott Laboratories, Chicago, Illinois, USA) and heparin $(6 \mathrm{U} / \mathrm{h})$ were infused for 5 hours to raise circulating fatty acid concentrations and a 2-hour hyperinsulinemic-euglycemic clamp procedure followed as previously described (39). 
Biochemical assays and calculation. Plasma glucose, insulin, and fatty acid concentrations during the clamp procedure were analyzed as previously described (17). Plasma concentrations of $\left[3-{ }^{3} \mathrm{H}\right]$ glucose, 2- $\left[{ }^{14} \mathrm{C}\right] \mathrm{DG}$, and ${ }^{3} \mathrm{H}_{2} \mathrm{O}$ were determined following deproteinization of plasma samples as previously described (17). The radioactivity of ${ }^{3} \mathrm{H}$ in tissue glycogen was determined by digesting tissue samples in $\mathrm{KOH}$ and precipitating glycogen with ethanol. For the determination of tissue 2-[14C]DG-6-phosphate (2-DG-6-P) content, tissue samples were homogenized, and the supernatants were subjected to an ion-exchange column to separate 2- $\left[{ }^{14} \mathrm{C}\right] \mathrm{DG}-6-\mathrm{P}$ from $2-\left[{ }^{14} \mathrm{C}\right] \mathrm{DG}$.

Rates of basal and insulin-stimulated whole-body glucose turnover were determined as the ratio of the $\left[{ }^{3} \mathrm{H}\right]$ glucose infusion rate (disintegrations per minute) to the specific activity of plasma glucose (disintegrations per minute per micromole) at the end of basal period and during the final 30 minutes of the clamp procedure, respectively. Hepatic glucose production during the hyperinsulinemic-euglycemic clamps was determined by subtracting the glucose infusion rate from the whole-body glucose uptake. Whole-body glycolysis and glycogen plus lipid synthesis from glucose were calculated as previously described (17). Glucose uptake in individual tissues was calculated from a plasma $2-\left[{ }^{14} \mathrm{C}\right] \mathrm{DG}$ profile, which was fitted with a double exponential or linear curve using MLAB (Civilized Software Inc., Silver Springs, Maryland, USA) and tissue 2-[14C]DG-6-P content. Skeletal muscle glycolysis and glycogen synthesis were calculated as previously described (17).

Insulin signaling analysis. Skeletal muscle samples (gastrocnemius) were obtained at the end of the clamp procedure to measure in vivo activities of insulin receptor substrate-1 (IRS-1) tyrosine phosphorylation and IRS-1-associated PI3K. The activities were assessed by immunoprecipitating IRS-1 using antibodies to IRS-1 (Upstate Biotechnology Inc., Lake Placid, New York, USA) and assessing the incorporation of ${ }^{32} \mathrm{P}$ into PI to yield phosphatidylinositol-3monophosphate as previously described (17).

Measurement of fatty acid-derived metabolites in skeletal muscle and liver. Skeletal muscle (quadriceps) and livers of WT and FATP1 KO mice were studied to determine the concentrations of intracellular fatty acid-derived metabolites (i.e., fatty acyl-CoA, diacylglycerol, and ceramide) using an API 3000 LC/MS/MS (PE Sciex, Foster City, California, USA) interfaced with TurboIonSpray ionization source and using the modified method of Bligh and colleagues (40).

Statistical analysis. Data are expressed as means plus or minus SE. The significance of the difference in mean values of WT mice fed normal chow diet (control) versus WT mice fed a high-fat diet, WT mice with lipid infusion, FATP1 KO mice fed normal chow diet, FATP1 KO mice fed a high-fat diet, and FATP1 KO mice with lipid infusion was evaluated using the Duncan multiple range test.

\section{Results}

Generation of FATP1 KO mice. FATP1 KO mice were generated by replacing the first coding exon of FATP1, which encodes the amino acids $1-56$, with a targeting cassette using homologous recombination (Figure 1A). FATP1-deletion mice were obtained at Mendelian ratios from heterozygous mating pairs and appeared to be indistinguishable from WT animals. Southern blot analysis of tail DNA from $\mathrm{F}_{2}$-generation mice using $5^{\prime}$ and $3^{\prime}$ diagnostic probes, as well as PCR genotyping analysis, confirmed the correct recombination event (Figure 1, B and C). Analysis of FATP1 mRNA using real-time RT-PCR with a probe directed to the $3^{\prime}$ untranslated region of FATP1 showed that FATP1 mRNA was greatly reduced in the skeletal muscle and white adipose tissue of FATP1 KO mice (Figure 1D). We also com- pared gene expression in muscle and white adipose tissue of the WT and FATP1 KO mice using Affymetrix GeneChip analysis. Expression of FATP1 was markedly reduced in the skeletal muscle and white adipose tissue of FATP1 KO mice as compared with the WT mice, whereas expression of other FATPs (i.e., FATP2, FATP3, FATP4, and FATP5) did not differ among groups (data not shown). This demonstrates that the targeted disruption resulted in deletion of the functional FATP1 gene with no compensatory upregulation of other FATP family members. Moreover, expression of other genes associated with fatty acid uptake and metabolism (i.e., FATPs, CD36, ACS1) and de novo lipogenesis (i.e., fatty acid synthase) did not differ among groups, whereas FABP3 expression was slightly but significantly elevated in the FATP1 KO mice (5,306 \pm 240 versus 3,479 \pm 215 arbitrary units in skeletal muscle of WT mice). Expression of fatty acid oxidation-associated genes (i.e., acyl-CoA oxidase, carnitine palmitoyltransferase family members, long-chain acyl-CoA dehydrogenase) also did not differ among groups. Taken together, these findings suggest that little or no compensatory upregulation of lipid metabolic pathways occurred in the FATP1 KO mice.

Metabolic phenotypes of FATP1 KO mice. To evaluate the effect of FATP1 deletion on whole-body physiology, we determined body and organ weights as well as plasma parameters from WT and FATP1 KO mice fed a normal chow diet or a high-fat diet. Body weights, fat pad weights, and other tissue weights were similar in the male FATP1 KO and WT mice fed a normal chow diet or high-fat diet. Circulating levels of glucose, insulin, fatty acids, and triglycerides did not significantly differ among groups, while none of these parameters differed between male and female mice (data not shown). Furthermore, whole-body lean and fat mass did not differ between WT and FATP1 KO mice on a normal chow diet, and high-fat feeding similarly increased whole-body fat mass in the WT and FATP1 KO mice. We

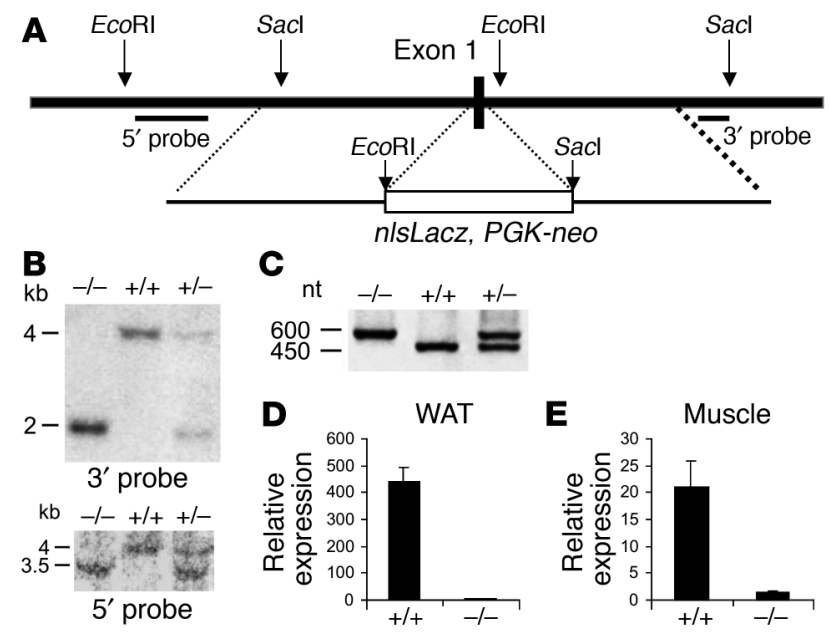

\section{Figure 1}

Generation of FATP1 KO mice. (A) Map of the FATP1 genomic locus and the targeting constructs. The $5^{\prime}$ and $3^{\prime}$ probes that were used to verify the homologous recombination event are indicated. (B) Southern blot using genomic DNA from the tail of $F_{2}$ animals digested with EcoRI and Sacl and hybridized with the $5^{\prime}$ and $3^{\prime}$ probes, respectively. The size of $\mathrm{KO}$ and WT alleles is indicated. (C) DNA products obtained by PCR amplification using the genotyping primers described in Methods and genomic DNA from the tail of $F_{2}$ animals as a template. nt, nucleotide. (D and E) FATP1 mRNA levels in white adipose tissue (WAT) and skeletal muscle of KO and WT animals. 
Table 1

Metabolic parameters during basal (overnight-fasted) and hyperinsulinemic-euglycemic clamp periods in the WT and FATP1 K0 mice with or without lipid infusion or high-fat feeding

\begin{tabular}{|c|c|c|c|c|c|c|c|c|}
\hline & \multirow[b]{2}{*}{$n$} & \multirow[b]{2}{*}{$\begin{array}{c}\text { Body wt } \\
\text { (g) }\end{array}$} & \multicolumn{3}{|c|}{ Basal period } & \multicolumn{3}{|c|}{ Clamp period } \\
\hline & & & $\begin{array}{c}\text { Plasma } \\
\text { glucose (mM) }\end{array}$ & $\begin{array}{c}\text { Plasma } \\
\text { insulin (pM) }\end{array}$ & $\begin{array}{l}\text { Plasma fatty } \\
\text { acids (mM) }\end{array}$ & $\begin{array}{c}\text { Plasma } \\
\text { glucose }(\mathrm{mM})\end{array}$ & $\begin{array}{c}\text { Plasma } \\
\text { insulin (pM) }\end{array}$ & $\begin{array}{l}\text { Plasma fatty } \\
\text { acids (mM) }\end{array}$ \\
\hline WT & 6 & $30 \pm 1$ & $7.9 \pm 0.7$ & $130 \pm 15$ & $0.3 \pm 0.1$ & $6.6 \pm 0.6$ & $409 \pm 22$ & $0.03 \pm 0.01$ \\
\hline FATP1 K0 & 6 & $28 \pm 1$ & $7.2 \pm 0.4$ & $105 \pm 14$ & $0.2 \pm 0.1$ & $6.6 \pm 0.5$ & $455 \pm 36$ & $0.07 \pm 0.02$ \\
\hline WT lipid infused & 5 & $30 \pm 2$ & $7.1 \pm 0.6$ & $119 \pm 29$ & $1.6 \pm 0.2^{\mathrm{A}}$ & $6.6 \pm 0.7$ & $432 \pm 52$ & $1.77 \pm 0.26^{\mathrm{A}}$ \\
\hline FATP1 KO lipid infused & 5 & $27 \pm 2$ & $6.2 \pm 0.4$ & $86 \pm 10$ & $1.7 \pm 0.1^{\mathrm{A}}$ & $7.1 \pm 0.9$ & $420 \pm 16$ & $1.88 \pm 0.11^{\mathrm{A}}$ \\
\hline WT high-fat diet & 5 & $37 \pm 1^{A}$ & $10.2 \pm 1.6$ & $110 \pm 18$ & $1.2 \pm 0.1^{\mathrm{A}}$ & $6.9 \pm 1.2$ & $427 \pm 56$ & ND \\
\hline FATP1 KO high-fat diet & 5 & $37 \pm 2^{A}$ & $8.4 \pm 0.7$ & $92 \pm 15$ & $1.2 \pm 0.1^{\mathrm{A}}$ & $7.3 \pm 0.6$ & $442 \pm 61$ & ND \\
\hline
\end{tabular}

AP $<0.05$ versus WT mice by Duncan multiple range test. ND, not determined.

also did not observe any differences in muscle and heart histology using samples from 8-week-old and 15-month-old male mice maintained on a chow diet. Since adipose tissue-derived hormones have been shown to alter glucose homeostasis, we measured expression of adiponectin and resistin in the white adipose tissue of mice using real-time RT-PCR. While adiponectin expression did not differ among groups, the expression of resistin was significantly lower in the FATP1 KO mice on a normal chow diet $(2.5 \pm 0.4$ versus $8.0 \pm 2.0$ arbitrary units in the WT mice), but not on the high-fat diet. Furthermore, fatty acid uptake in skeletal muscle was measured in vitro using ${ }^{3} \mathrm{H}$-laurate incubation with isolated soleus muscles from chow-fed mice and found to be unaltered in the FATP1 KO mice as compared with the WT mice (data not shown). The in vitro assay conditions used may not have been suitable to detect the contribution of FATP1 to muscle fatty acid uptake; alternatively, FATP1 may be required for uptake only under certain physiological conditions.

Hyperinsulinemic-euglycemic clamp experiments in FATP1 KO mice. Insulin action on glucose uptake and metabolism was examined during a 2-hour hyperinsulinemic-euglycemic clamp procedure in awake, age-matched, male WT and FATP1 KO mice following a 5-hour lipid infusion or a 3 -week feeding of a high-fat diet. Overnight-fasted plasma glucose concentrations did not differ between WT and FATP1 KO mice fed a regular chow diet, but showed a tendency to increase in the WT mice following the highfat diet (Table 1). During the clamp procedures, plasma insulin concentration was raised to approximately $450 \mathrm{pM}$, while the plasma glucose concentration was maintained at approximately $7 \mathrm{mM}$ in all groups (Table 1). Plasma fatty acid concentrations were elevated approximately twofold to threefold following a 5-hour lipid infusion or 3 weeks of high-fat feeding in the WT and FATP1 KO mice (Table 1). Plasma fatty acid concentrations were completely suppressed during clamp procedures in both groups, but remained elevated during clamp procedures in the lipid-infused groups.

The glucose infusion rate required to maintain euglycemia increased rapidly in the WT and FATP1 KO mice fed a normal chow diet and reached a steady state within 90 minutes. In contrast, there was a markedly blunted insulin response during the hyperinsulinemic-euglycemic clamp studies in the WT mice after lipid infusion or high-fat feeding, as reflected by a much lower steady-state glucose infusion rate (Figure 2A). In contrast, lipid infusion or a high-fat diet did not affect the steady-state glucose infusion rate in the FATP1 KO mice (Figure 2A). Insulin-stimulated whole-body glucose turnover was decreased by approximately $40 \%$ in the WT mice following lipid infusion or high-fat diet, whereas it remained normal in the FATP1 KO mice (Figure 2B). Tissue-specific insulin-stimulated glucose uptake in vivo was estimated using administration of $2-\left[{ }^{14} \mathrm{C}\right] \mathrm{DG}$, a nonmetabolizable glucose analogue, during clamp procedures in awake mice. Lipid infusion or high-fat feeding decreased insulin-stimulated glucose uptake in skeletal muscle (gastrocnemius) of the WT mice by approximately $70 \%$. In contrast, FATP1 KO mice were protected from fat-induced decreases in insulin-stimulated glucose uptake in skeletal muscle (Figure $2 \mathrm{C}$ ). In contrast to the protective effects of FATP1 deletion on fat-induced insulin resistance in skeletal muscle, insulin-stimulated glucose uptake in brown adipose tissue was significantly reduced following lipid infusion or high-fat feeding in both groups (Figure 2D). Insulin-stimulated glucose uptake in white adipose tissue was neither affected by fat challenge nor FATP1 deletion (data not shown).
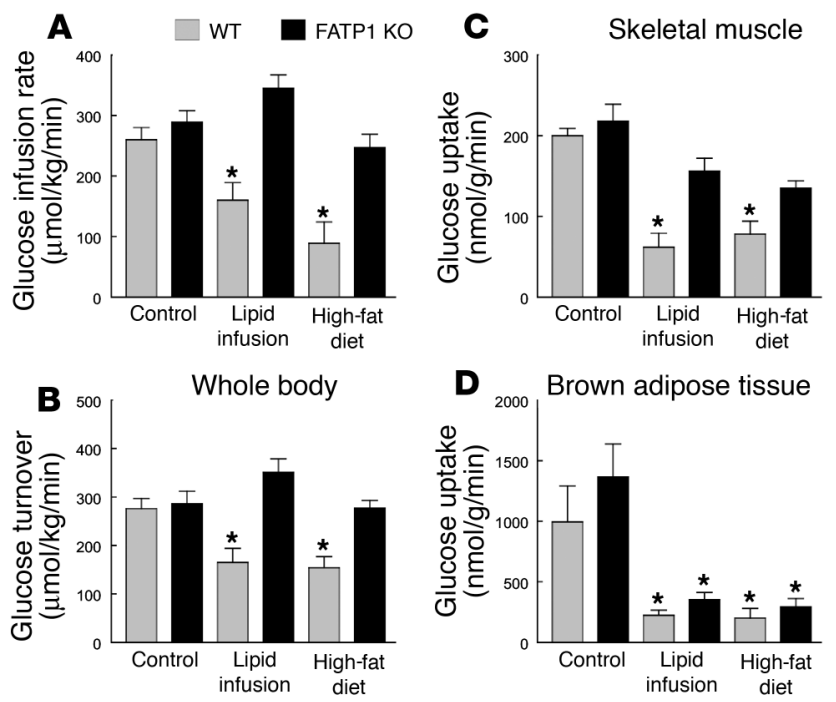

\section{Figure 2}

Whole-body and tissue-specific glucose uptake in WT (gray bars) and FATP1 KO (black bars) mice with lipid infusion or high-fat feeding. (A) Steady-state glucose infusion rate, obtained from averaged rates of 90 to 120 minutes of hyperinsulinemic-euglycemic clamps. (B) Insulin-stimulated whole-body glucose turnover in vivo. (C) Insulin-stimulated skeletal muscle glucose uptake in vivo. (D) Insulin-stimulated glucose uptake in brown adipose tissue in vivo. Values are means \pm SE for 5-6 experiments. ${ }^{*} P<0.05$ versus WT (control) mice. 

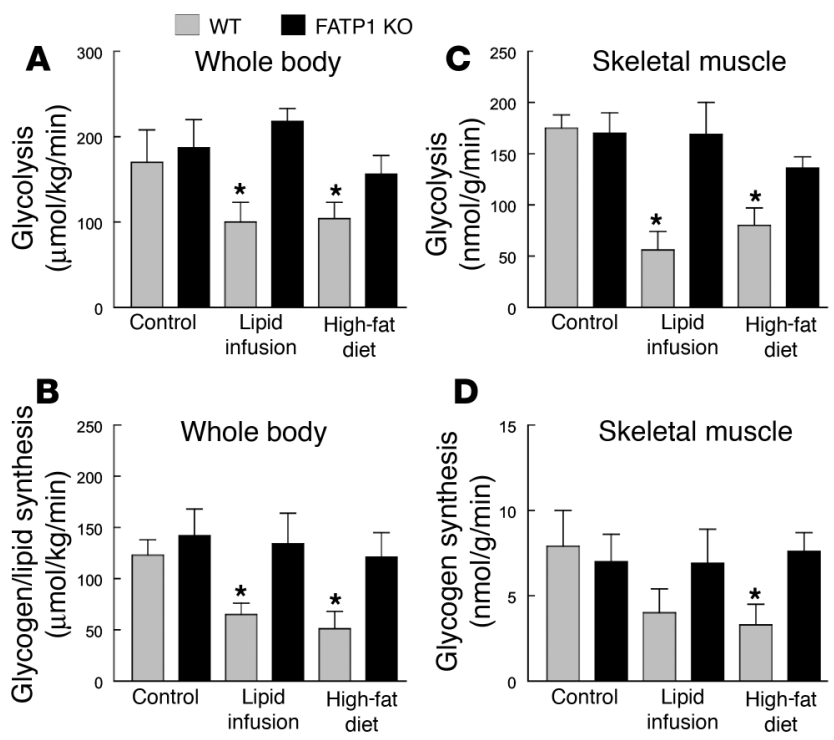

Figure 3

Insulin-stimulated whole-body and skeletal muscle (gastrocnemius) glucose metabolic flux in WT (gray bars) and FATP1 KO (black bars) mice with lipid infusion or high-fat feeding. (A) Insulin-stimulated whole-body glycolysis in vivo. (B) Insulin-stimulated whole-body glycogen plus lipid synthesis from glucose in vivo. (C) Insulin-stimulated skeletal muscle glycolysis in vivo. (D) Insulin-stimulated skeletal muscle glycogen synthesis in vivo. Values are means \pm SE for $5-6$ experiments. ${ }^{*} P<0.05$ versus WT (control) mice.

Insulin-stimulated whole-body and skeletal muscle glucose metabolic flux were measured using systemic appearance of ${ }^{3} \mathrm{H}_{2} \mathrm{O}$ as a by-product of $\left[{ }^{3} \mathrm{H}\right]$ glucose metabolism and $\left[{ }^{3} \mathrm{H}\right]$ glucose incorporation into muscle glycogen, respectively. Whole-body glycolysis and glycogen plus lipid synthesis from glucose were decreased by approximately $50 \%$ in the WT mice following lipid infusion or highfat feeding (Figure 3, A and B). In contrast, lipid infusion or highfat feeding did not affect insulin-stimulated whole-body glucose metabolism in the FATP1 KO mice. Furthermore, insulin-stimulated glycolysis and glycogen synthesis in skeletal muscle (gastrocnemius) were decreased by approximately $80 \%$ in the WT mice following lipid infusion or high-fat feeding (Figure 3, C and D). In contrast, FATP1 KO mice were protected from fat-induced decreases in insulin-stimulated muscle glucose metabolism. These results indicate an important role of FATP1 and fatty acid uptake in skeletal muscle in the development of fat-induced insulin resistance. In contrast to the effects on peripheral glucose metabolism, neither fat challenge nor FATP1 deletion affected basal hepatic glucose production (HGP; Figure 4C). Additionally, insulin's ability to suppress basal HGP was similarly reduced following high-fat feeding in the WT and FATP1 KO mice, as demonstrated by significantly elevated HGP during an insulin-stimulated state (i.e., clamps) in the WT mice as well as an increasing tendency for elevated HGP in the FATP1 KO mice (Figure 4D).

Skeletal muscle insulin signaling in FATP1 KO mice. Recent studies in IRS-1 gene-disrupted mice have suggested that IRS- 1 is important in insulin activation of glucose transport and glycogen synthase activity in skeletal muscle $(2,41)$. Insulin-stimulated IRS-1 tyrosine phosphorylation in skeletal muscle (gastrocnemius) was decreased by approximately $60 \%$ in the WT mice following lipid infusion or high-fat feeding (Figure 4A). Similarly, insulin-stimulated IRS-1-associated PI3K activity in skeletal muscle (gastrocnemius) was decreased by $40 \%$ and $75 \%$ in the WT mice following lipid infusion and high-fat feeding, respectively (Figure 4B). In contrast, FATP1 KO mice were protected from fat-induced defects in insulin-stimulated IRS-1 tyrosine phosphorylation and IRS-1associated PI3K activity in skeletal muscle (Figure 4, A and B).

Intramuscular fat metabolite contents in FATP1 KO mice. Defects in skeletal muscle insulin action following lipid infusion or a high-fat diet may be due to increases in intramuscular fatty acid-derived metabolites. Previous studies have shown a strong inverse relationship between intramuscular fat content and insulin sensitivity in humans and animals (15-19). To examine whether the fat-induced changes in skeletal muscle insulin action were associated with changes in intramuscular fat contents, intramuscular concentrations of triglyceride and fatty acid-derived metabolites (i.e., fatty acyl-CoA, diacylglycerol, ceramide) were measured in skeletal muscle and liver using liquid chromatography tandem spectrometry. Fatty acid composition of lipid emulsion and high-fat diet was also determined, and the results indicated that $\mathrm{C} 16, \mathrm{C} 18: 1$, and $\mathrm{C} 18: 2$ were the more abundant fatty acyl-CoAs in the lipid emulsion and high-fat diet (Figure $5 A$ ). Intramuscular (quadriceps) triglyceride concentration was increased in the WT mice following a high-fat diet but remained normal in the FATP1 KO mice (Figure 5B). Similarly, intramuscular total fatty acyl-CoA concentrations were significantly elevated in the WT mice following lipid infusion or a high-fat diet, whereas FATP1 KO mice were completely protected from fat-induced increases in intramuscular fatty acyl-CoA levels (Figure 6A). Fatty acid composition of muscle fatty acyl-CoA further indicates that increases in C18:1 and C18:2 were most dramatic following acute lipid infusion and highfat feeding, and these levels were markedly lower in the FATP1 KO mice. These findings also indicate the role of FATP1 as acyl-CoA syn-
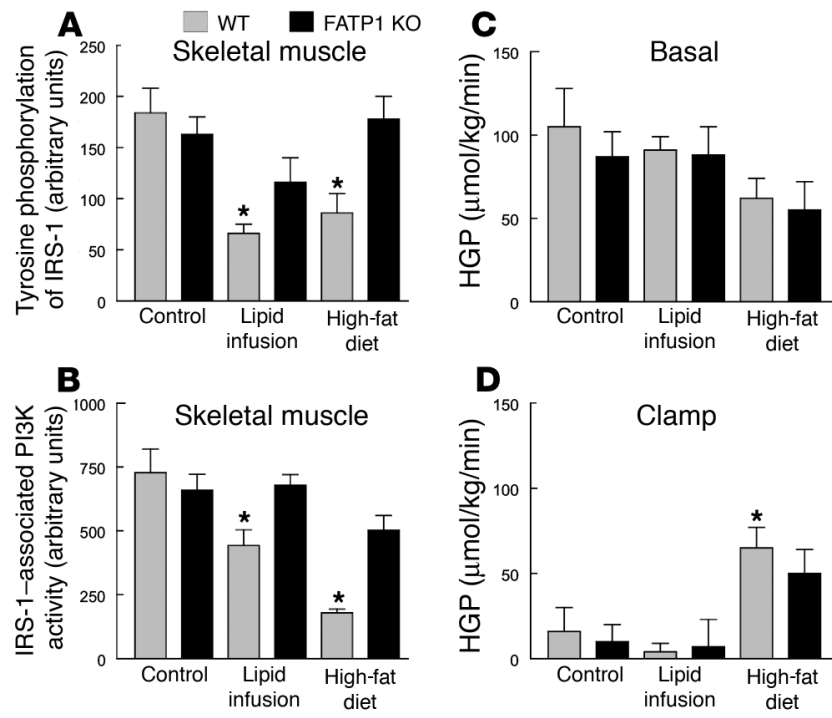

\section{Figure 4}

Insulin-signaling measurement in skeletal muscle (gastrocnemius) and hepatic insulin action of the WT (gray bars) and FATP1 KO (black bars) mice with lipid infusion or high-fat feeding. (A) Insulin-stimulated tyrosine phosphorylation of IRS-1 in skeletal muscle. (B) Insulin-stimulated IRS-1-associated PI3K activity in skeletal muscle. (C) Basal HGP. (D) HGP during hyperinsulinemic-euglycemic clamp procedures. Values are means \pm SE for $5-6$ experiments. ${ }^{*} P<0.05$ versus WT (control) mice. 

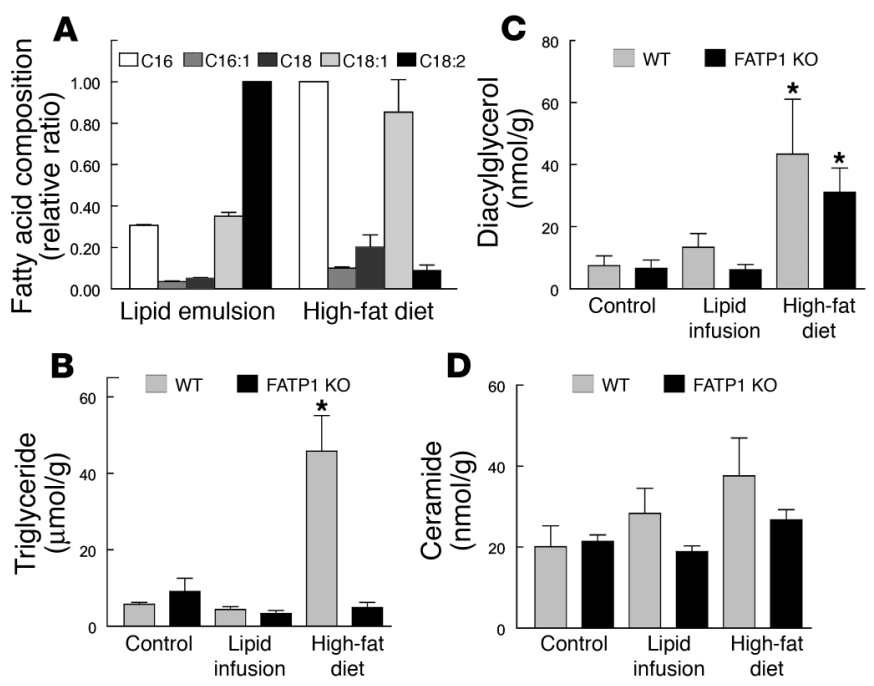

Figure 5

Fatty acid composition and intramuscular (quadriceps) fat contents in WT (gray bars) and FATP1 KO (black bars) mice with lipid infusion or highfat feeding. (A) Fatty acid composition of high-fat diet and lipid emulsion administered in in vivo experiments. (B) Intramuscular triglyceride concentrations. (C) Intramuscular diacylglycerol concentrations. (D) Intramuscular ceramide concentrations. Values are mean \pm SE for 5-6 experiments. ${ }^{\star} P<0.05$ versus WT (control) mice.

thetase for long chain and very long chain fatty acids. Although intramuscular diacylglycerol and ceramide concentrations showed a similar pattern of changes following lipid infusion or a high-fat diet, the changes were not as significant or apparent as those of fatty acyl-CoA levels (Figure 5, C and D). In contrast, intrahepatic total fatty acylCoA levels were not significantly altered among groups (Figure 6B).

\section{Discussion}

The mechanism by which increased levels of fatty acid-derived metabolites cause skeletal muscle insulin resistance involves activation of a serine kinase cascade, of which PKC- $\theta$ and/or IKB kinase- $\beta$ (IKK- $\beta$ ) may play a role, leading to the serine phosphorylation of IRS-1 (39, 42-44). Recent studies have shown that serine phosphorylation of IRS- 1 prevents tyrosine phosphorylation of IRS- 1 and interferes with its ability to activate PI3K, leading to insulin resistance, as occurs upon treatment with TNF- $\alpha$ and okadaic acid (45, 46). In support of this hypothesis, we have recently demonstrated that skeletal muscle insulin resistance induced by 5 -hour lipid infusion was due to increases in intramuscular fatty acyl-CoA and diacylglycerol levels, activation of PKC- $\theta$, and subsequent defects in insulin signaling activities (21). In this regard, fatty acyl-CoA and diacylglycerol are potent activators of PKC- $\theta(47,48)$, while Itani and colleagues have shown increased activity of PKC in obese insulin resistant subjects (42). Additionally, Yuan and colleagues have demonstrated that activation of IKK- $\beta$ resulted in defects in skeletal muscle insulin signaling (43), while our previous study has shown that mice with heterozygous deletion of IKK- $\beta$ were protected from fat-induced insulin resistance in skeletal muscle (39).

Since FATP1 is an acyl-CoA synthetase that can mediate fatty acid uptake in vitro, we examined whether FATP1 deletion could protect mice from fat-induced insulin resistance. Our findings demonstrated that FATP1 KO mice were protected from chronic diet-induced or acute lipid infusion-induced increases in intramuscular fatty acid metabolites, and this was associated with protection from fatinduced defects in insulin signaling activities and insulin action in skeletal muscle. These results support the notion that FATP1 plays an important role in fatty acid uptake and acyl-CoA synthesis in skeletal muscle, and they are the first demonstration of a role of FATP1 on fatty acid metabolism and glucose homeostasis in vivo. Our findings further support the hypothesis that fat-induced insulin resistance in skeletal muscle involves intracellular accumulation of fatty acid-derived metabolites and subsequent defects in IRS-1-associated PI3K activity and glucose transport activity. Since recent studies have implicated circulating factors released by adipocytes, in particular resistin and adiponectin, in playing a role in skeletal muscle insulin resistance (49-54), we examined this alternative mechanism of insulin resistance. Adipose tissue expression of resistin and adiponectin was not significantly altered following high-fat feeding in the FATP1 KO mice as compared with the WT mice, suggesting that dysregulation of these factors did not play a major role in the observed changes in insulin sensitivity. While we cannot exclude the possibility that other adipose-derived factors are involved in the FATP1 phenotype, the lack of an overt effect of FATP1 deletion on adipose tissue mass and gene regulation make an indirect effect less likely. Thus, our present findings that FATP1 KO mice were protected from fat-induced increases in intramuscular fatty acyl-CoA and insulin resistance demonstrate an important role for intramuscular fatty acid metabolites as causative agents in the development of insulin resistance in skeletal muscle.
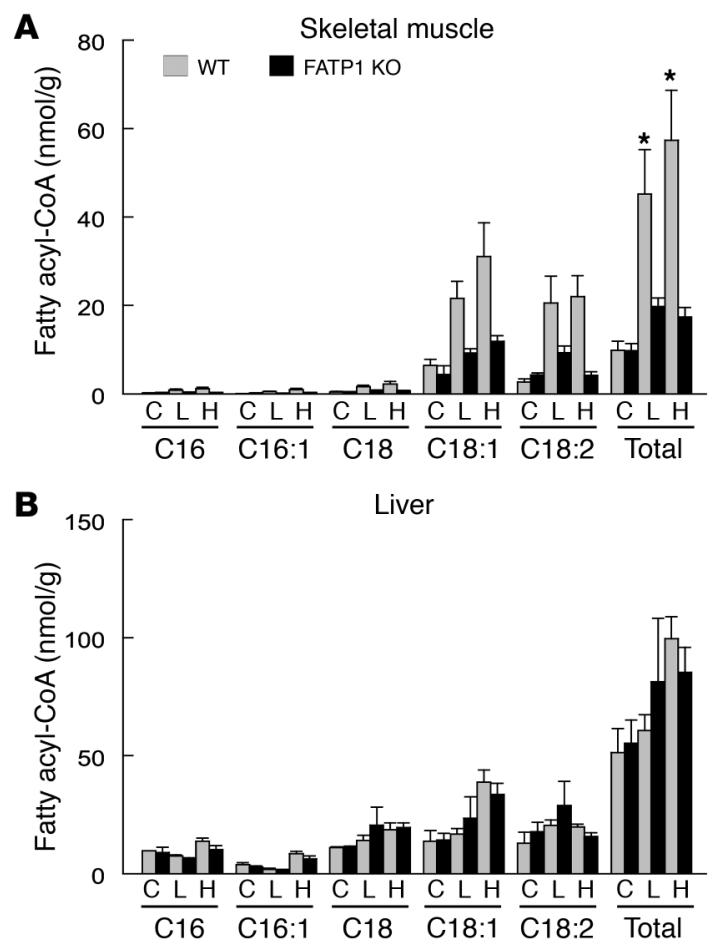

Figure 6

Fatty acid composition of intramuscular (quadriceps) and intrahepatic fatty acyl-CoAs of the WT (gray bars) and FATP1 KO (black bars) mice with control $(C)$, lipid infusion $(L)$, or high-fat feeding $(H)$. (A) Skeletal muscle fatty acyl-CoAs. (B) Liver fatty acyl-CoAs. Values are means \pm SE for 5-6 experiments. ${ }^{*} P<0.05$ versus FATP1 KO mice in total fatty acyl-CoA levels. 
While the protection from fat-induced insulin resistance in skeletal muscle of FATP1 KO mice is consistent with the in vitro data on FATP1 function and the proposed mechanisms of fat-induced insulin resistance, there were several interesting aspects of the FATP1 KO phenotype. First, FATP1 KO mice were phenotypically indistinguishable from the WT mice when both groups were fed a regular chow diet. In particular, intramuscular fatty acid metabolite levels and skeletal muscle insulin action were similar in the WT and FATP1 KO mice fed a regular chow diet. This finding is in marked contrast to the observation of enhanced insulin sensitivity in the CD36 KO mice, which showed increased glucose utilization and fasting hypoglycemia (20). Additionally, circulating levels of fatty acids and triglyceride were similar in the WT and FATP1 KO mice fed either a regular chow diet or a high-fat diet, and this observation was also in contrast to increased plasma fatty acids levels, due to reduced fatty acid clearance, in the CD36-null mice (20). Moreover, despite complete ablation of FATP1 in adipose tissue, FATP1 KO mice gained similar adiposity on a regular chow or a high-fat diet as compared with the WT mice. FATP1 deletion also did not affect glucose metabolism in the heart (795 \pm 100 versus $840 \pm 181 \mathrm{nmol} / \mathrm{g} / \mathrm{min}$ in the WT mice). Taken together, these observations indicate that FATP1 may mediate the uptake of fatty acids under particular physiological conditions (e.g., increased lipid supply) and/or uptake of a subset of fatty acids, possibly channeling them to a particular metabolic fate.

Overall, our findings show that FATP1 KO mice are protected from fat-induced accumulation of intramuscular fatty acyl-CoA and insulin resistance in skeletal muscle. These results support the notion that intramuscular fatty acid-derived metabolites are the major causative factor responsible for insulin resistance in skeletal muscle. Our data also show an important role for FATP1 in the conversion of plasma free fatty acids to intramuscular acyl-CoA, demon- strating that FATP1 is required for uptake of fatty acids under particular conditions. FATP1 may play an important role in the pathophysiology of type 2 diabetes and may constitute a novel therapeutic target for its treatment.

\section{Acknowledgments}

Part of this study was conducted at the Yale Mouse Metabolic Phenotyping Center and was supported by grants from the U.S. Public Health Service: U24 DK-59635 (to G.I. Shulman and J.K. Kim); R01 DK-40936 and P30 DK-45735 (to G.I. Shulman); and P01 HL6610501 and R37 DK47618-13 (to H.F. Lodish). This study was also supported by a grant from the American Diabetes Association (7-01-JF05 to J.K. Kim). Gerald I. Shulman is an Investigator of the Howard Hughes Medical Institute. We are grateful to Anthony Romanelli and Aida Groszmann for technical assistance, Ann Strauss and Victoria Fairchild-Huntress for help with generating the KO animals, and Dennis Huszar for advice and discussion.

Received for publication May 14, 2003, and accepted in revised form January 6, 2004.

Address correspondence to: Jason K. Kim, Yale University School of Medicine, Department of Internal Medicine, Section of Endocrinology and Metabolism, The Anlyan Center, South 269C, PO Box 208020, New Haven, Connecticut 06520-8020, USA. Phone: (203) 785-6716; Fax: (203) 785-6753; E-mail: jason.k.kim@yale.edu.

Jason K. Kim and Ruth E. Gimeno contributed equally to this work.

Ruth E. Gimeno's present address is: Wyeth Research, Cambridge, Massachusetts, USA.
1. Shaw, J.E., Zimmet, P.Z., McCarty, D, and de Courten, M. 2000. Type 2 diabetes worldwide according to the new classification and criteria. Diabetes Care. 23(Suppl. 2):B5-B10.

2. Kahn, C.R. 1994. Insulin action, diabetogenes, and the cause of type II diabetes. Diabetes. 43:1066-1084.

3. DeFronzo, R.A. 1988. The triumvirate: beta-cell, muscle, liver. A collusion responsible for NIDDM. Diabetes. 37:667-687.

4. Cline, G.W., et al. 1999. Impaired glucose transport as a cause of decreased insulin-stimulated muscle glycogen synthesis in type 2 diabetes. N. Engl.J. Med. 341:240-246.

5. Hotamisligil, G.S., Shargill, N.S., and Spiegelman, B.M. 1993. Adipose expression of tumor necrosis factor-alpha: direct role in obesity-linked insulin resistance. Science. 259:87-91.

6. Steppan, C.M., et al. 2001. The hormone resistin links obesity to diabetes. Nature. 409:307-312.

7. Yamauchi, T., et al. 2001. The fat-derived hormone adiponectin reverses insulin resistance associated with both lipoatrophy and obesity. Nat. Med. 7:941-946.

8. Hotta, K., et al. 2000. Plasma concentrations of a novel, adipose-specific protein, adiponectin, in type 2 diabetic patients. Arterioscler. Thromb. Vasc. Biol. 20:1595-1599.

9. Kahn, C.R., Chen, L., and Cohen, S.E. 2000. Unraveling the mechanism of action of thiazolidinediones. J. Clin. Invest. 106:1305-1307.

10. Randle, P.J., Garland, P.B., Hales, C.N., and Newsholme, E.A. 1963. The glucose fatty acid cycle: its role in insulin sensitivity and the metabolic disturbances of diabetes mellitus. Lancet. 1:785-789.

11. McGarry, J.D. 1992. What if Minkowski had been ageusic? An alternative angle on diabetes. Science. 258:766-770.
12. Reaven, G.M. 1988. Role of insulin resistance in human disease. Diabetes. 37:1595-1607.

13. Boden, G. 2001. Obesity, free fatty acids, and insulin resistance. Current Opinion in Endocrinology \& Diabetes. 8:235-239.

14. Shulman, G.I. 2000. Cellular mechanisms of insulin resistance. J. Clin. Invest. 106:171-176.

15. Kraegen, E.W., Cooney, G.J., Ye, J., and Thompson, A.L. 2001. Triglycerides, fatty acids and insulin resistance - hyperinsulinemia. Exp. Clin. Endocrinol. Diabetes. 109(Suppl.):S516-S526.

16. Boden, G., Lebed, B., Schatz, M., Homko, C., and Lemieux, S. 2001. Effects of acute changes of plasma free fatty acids on intramyocellular fat content and insulin resistance in healthy subjects. Diabetes. 50:1612-1617.

17. Kim, J.K., et al. 2001. Tissue-specific overexpression of lipoprotein lipase causes tissue-specific insulin resistance. Proc. Natl. Acad. Sci. U. S. A. 98:7522-7527.

18. Krssak, M., et al. 1999. Intramyocellular lipid concentrations are correlated with insulin sensitivity in humans: a ${ }^{1} \mathrm{H}$ NMR spectroscopy study. Diabetologia. 42:113-116.

19. Perseghin, G., et al. 1999. Intramyocellular triglyceride content is a determinant of in vivo insulin resistance in humans. $\mathrm{A}{ }^{1} \mathrm{H}-{ }^{13} \mathrm{C}$ nuclear magnetic resonance spectroscopy assessment in offspring of type 2 diabetic patients. Diabetes. 48:1600-1606.

20. Hajri, T., Han, X.X., Bonen, A., and Abumrad, N.A. 2002. Defective fatty acid uptake modulates insulin responsiveness and metabolic responses to diet in CD36-null mice. J. Clin. Invest. 109:1381-1389. doi:10.1172/JCI200214596.

21. Yu, C., et al. 2002. Mechanism by which fatty acids inhibit insulin activation of insulin receptor substrate-1 (IRS-1)-associated phosphatidylinositol 3-kinase activity in muscle. J. Biol. Chem.
277:50230-50236.

22. Schaffer, J.E., and Lodish H.F. 1994. Expression cloning and characterization of a novel adipocyte long chain fatty acid transport protein. Cell. 79:427-436.

23. Abumrad, N., Coburn, C., and Ibrahimi, A. 1999. Membrane proteins implicated in long-chain fatty acid uptake by mammalian cells: CD36, FATP and FABPm. Biochim. Biophys. Acta. 1441:4-13.

24. Bonen, A., Miskovic, D., and Kiens, B. 1999. Fatty acid transporters (FABPpm, FAT, FATP) in human muscle. Can. J. Appl. Physiol. 24:515-523.

25. Faergeman, N.J., DiRusso, C.C., Elberger, A., Knudsen, J., and Black, P.N. 1997. Disruption of the Saccharomyces cerevisiae homologue to the murine fatty acid transport protein impairs uptake and growth on long-chain fatty acids. J. Biol. Chem. 272:8531-8538.

26. Hall, A.M., Smith, A.J., and Bernlohr, D.A. 2003 Characterization of the acyl CoA synthetase activity of purified murine fatty acid transport protein 1 . J. Biol. Chem. 278:43008-43013.

27. Stuhlsatz-Krouper, S.M., Bennett, N.E., and Schaffer, J.E. 1998. Substitution of alanine for serine 250 in the murine fatty acid transport protein inhibits long chain fatty acid transport. J. Biol. Chem. 273:28642-28650.

28. Hatch, G.M., Smith, A.J., Xu, F.Y., Hall, A.M., and Bernlohr, D.A. 2002. FATP1 channels exogenous FA into 1,2,3-triacyl-sn-glycerol and downregulates sphingomyelin and cholesterol metabolism in growing 293 cells. J. Lipid Res. 43:1380-1389.

29. Stahl, A., Gimeno, R.E., Tartaglia, L.A., and Lodish, H.F. 2001. Fatty acid transport proteins: a current view of a growing family. Trends Endocrinol. Metab. 12:266-273.

30. Gimeno, R.E., et al. 2003. Targeted deletion of fatty 
acid transport protein-4 results in early embryonic lethality. J. Biol. Chem. 278:49512-49516.

31. Herrmann, T., et al. 2003. Mice with targeted disruption of the fatty acid transport protein 4 (FATP4, Slc27a4) gene show features of lethal restrictive dermopathy. J. Cell Biol. 161:1105-1115.

32. Stahl, A., Evans, J.G., Pattel, S., Hirsch, D., and Lodish, H.F. 2002. Insulin causes fatty acid transport protein translocation and enhanced fatty acid uptake in adipocytes. Dev. Cell. 2:477-488.

33. Gimeno, R.E., et al. 2003. Characterization of a heart-specific fatty acid transport protein. J. Biol. Chem. 278:16039-16044.

34. Coburn, C.T., et al. 2000. Defective uptake and utilization of long-chain fatty acids in muscle and adipose tissue of CD36 knockout mice. J. Biol. Chem. 275:32523-32529.

35. Binas, B., Danneberg, H., McWhir, J., Mullins, L., and Clark, A.J. 1999. Requirement for the heart-type fatty acid binding protein in cardiac fatty acid utilization. FASEB J. 13:805-812.

36. Zou, Z., et al. 2003. Vectorial acylation in Saccharomyces cerevisiae. J. Biol. Chem. 278:16414-16422.

37. Ibrahimi, A., et al. 1999. Muscle-specific overexpression of FAT/CD36 enhances fatty acid oxidation by contracting muscle, reduces plasma triglycerides and fatty acids, and increases plasma glucose and insulin. J. Biol. Chem. 274:26761-26766.

38. Coburn, C.T., Hajri, T., Ibrahimi, A., and Abumrad, N.A. 2001. Role of CD36 in membrane transport and utilization of long-chain fatty acids by different tissues. J. Mol. Neurosci. 16:117-121.

39. Kim, J.K., et al. 2001. Prevention of fat-induced insulin resistance by salicylate. J. Clin. Invest. 108:437-446. doi:10.1172/JCI200111559.

40. Bligh, E.G., and Dyer, W.J. 1959. A rapid method of total lipid extraction and purification. Can. J. Biochem. Physiol. 37:911-917.

41. Previs, S.F., Withers, D.J., Ren, J.-M., White, M.F., and Shulman, G.I. 2000. Contrasting effects of IRS-1 versus IRS- 2 gene disruption on carbohydrate and lipid metabolism in vivo. J. Biol. Chem. 275:38990-38994.

42. Itani, S.I., Zhou, Q., Pories, W.J., MacDonald, K.G., and Dohm, G.L. 2000. Involvement of protein kinase $\mathrm{C}$ in human skeletal muscle insulin resistance and obesity. Diabetes. 49:1353-1358.

43. Yuan, M., et al. 2001. Reversal of obesity- and dietinduced insulin resistance with salicylates or targeted disruption of IKK-beta. Science. 293:1673-1677.

44. Schmitz-Peiffer, C., et al. 1997. Alterations in the expression and cellular localization of protein kinase $\mathrm{C}$ isozymes epsilon and theta are associated with insulin resistance in skeletal muscle of the high-fat-fed rats. Diabetes. 46:169-178.

45. Rui, L., et al. 2001. Insulin/IGF-1 and TNF- $\alpha$ stimulate phosphorylation of IRS-1 at inhibitory $\mathrm{Ser}^{307}$ via distinct pathways. J. Clin. Invest. 107:181-189.

46. Pederson, T.M., Kramer, D.L., and Rondinone, C.M. 2001. Serine/threonine phosphorylation of IRS-1 triggers its degradation. Possible regulation by tyrosine phosphorylation. Diabetes. 50:24-31.
47. Griffin, M.E., et al. 1999. Free fatty acid-induced insulin resistance is associated with activation of protein kinase $\mathrm{C} \theta$ and alterations in the insulin signaling cascade. Diabetes. 48:1270-1274.

48. Chalkley, S.M., Hettiarachchi, M., Chisholm, D.J., and Kraegen, E.W. 1998. Five-hour fatty acid elevation increases muscle lipids and impairs glycogen synthesis in the rat. Metabolism. 47:1121-1126.

49. Hofmann, C., et al. 1994. Altered gene expression for tumor necrosis factor- $\alpha$ and its receptors during drug and dietary modulation of insulin resistance. Endocrinology. 134:264-270.

50. Hotamisligil, G.S., et al. 1996. IRS-1-mediated inhibition of insulin receptor tyrosine kinase activity in TNF- $\alpha$ - and obesity-induced insulin resistance. Science. 271:665-668.

51. Nagaev, I., and Smith, U. 2001. Insulin resistance and type 2 diabetes are not related to resistin expression in human fat cells or skeletal muscle. Biochem. Biophys. Res. Comm. 285:561-564.

52. Sentinelli, F., et al. 2002. Human resistin gene, obesity, and type 2 diabetes. Mutation analysis and population study. Diabetes. 51:860-862.

53. Arita, Y., et al. 1999. Paradoxical decrease of an adipose-specific protein, adiponectin, in obesity. Biochem. Biophys. Res. Commun. 257:79-83.

54. Hotta, K., et al. 2001. Circulating concentrations of adipocyte protein adiponectin are decreased in parallel with reduced insulin sensitivity during the progression to type 2 diabetes in rhesus monkeys. Diabetes. 50:1126-1133. 